\title{
The Effect of Fish Oil Supplementation on Heart rate in Healthy Danish Infants
}

\author{
LOTTE LAURITZEN, JEPPE H. CHRISTENSEN, CAMILLA T. DAMSGAARD, AND KIM F. MICHAELSEN \\ Department of Human Nutrition [L.L., C.T.D., K.F.M.], University of Copenhagen, 1958 Frederiksberg C, Denmark; Department of \\ Nephrology [J.H.C.], Aarhus University Hospital, 9100 Aalborg, Denmark
}

\begin{abstract}
Polyunsaturated $n-3$ fatty acids ( $n$-3PUFA) may improve brain development and prevent cardiovascular disease. Heart rhythm is autonomically controlled and among the affected cardiovascular risk markers in adults. The aim of the study was to examine whether fish oil supplementation in late infancy could modify heart rate $(\mathrm{HR})$ and heart rate variability (HRV). In a $2 \times 2$-intervention, 83 healthy Danish infants were randomized to \pm fish oil $(3.4 \pm 1.1$ $\mathrm{mL} / \mathrm{d}$ ) and cow's milk or infant formula from 9 to 12 mo of age. In 57 infants, 0.5-h ECG recordings were successfully obtained before and after the intervention and erythrocyte fatty acid composition was determined in 30 of these. Fish oil supplementation raised erythrocyte $n$-3PUFA content $(p<0.001)$. No significant group differences were seen in HR or HRV. However, a fish-oil $\times$ gender interaction was observed on mean RR interval ( $p=0.001)$ with a $6 \%$ longer mean RR interval in fish-oil-supplemented boys ( $p=0.007)$. Irrespective of gender, there was a positive association between the 9and 12-mo changes in RR interval and erythrocyte $n$-3PUFA $(p<$ $0.001)$. In infants with confirmed changes in erythrocyte $n$-3PUFA, mean RR interval was found to be longer $(p=0.011)$ in the fish-oil-supplemented groups. The study suggests that fish oil may affect heart rhythm in infants similar to that observed in adults. This may imply low $n$-3PUFA-status in late infancy and $n$-3PUFA influence on CNS function. (Pediatr Res 64: 610-614, 2008)
\end{abstract}

$\mathrm{M}$ arine $n-3$ polyunsaturated fatty acids (n-3PUFA) are believed to be beneficial for CNS development (1) and the prevention of cardiovascular disease. Among the affected cardiovascular risk factors in adults are blood pressure (2) and heart rate (HR) (3) which are both influenced by the autonomic nervous system. Early intake of $n$-3PUFA is hypothesized to have programming effects on later cardiovascular health $(4,5)$. Perinatal maternal $n$-3PUFA deficiency has been shown to result in a life-long increase in blood pressure in rat offspring (6). In a randomized controlled trial with formulafed human infants, intake of long-chain polyunsaturated fatty acids of both the $n-3$ and $n-6$ type was also found to be inversely associated with blood pressure later in childhood (7).

In early infancy, $n$-3PUFA are supplied, as all other nutrients, by breast milk, which contains both short- and longchain $n$-3PUFA (1). After cessation of breast feeding, marine foodstuffs are the major source of long-chain $n$-3 polyunsat-

Received April 15, 2008; accepted July 7, 2008.

Correspondence: Lotte Lauritzen, Ph.D., Department of Human Nutrition, Faculty of Life Sciences, University of Copenhagen, Rolighedsvej 30, DK-1958 Frederiksberg C, Denmark; e-mail: 11@life.ku.dk

Supported by The Danish Research and Development Programme for Food and Technology. urated fatty acids (n-3LCPUFA), primarily eicosapentaenoic acid (EPA) and docosahexaenoic acid (DHA). Late infancy is a vulnerable period of rapid growth and transition from a milk-based diet to the family diet and the consumption of $n$-3LCPUFA is often limited during the complementary feeding period. In many countries, fish is not used as a common complementary food, and some countries even have an unsubstantiated recommendation to avoid the introduction of fish during infancy because of a believed increase in the risk of allergy (8). A daily supplement of cod liver oil during infancy and early childhood was previously recommended in the Nordic countries because of the content of vitamin D and A, and is still recommended in Norway, also because of the content of $n$-3LCPUFA. However, the implication of infant fish oil supplementation for health and development has not been thoroughly investigated.

HR and heart rate variability (HRV) was shown to be improved by fish oil supplementation in a number of randomized controlled trials in adults (9) and are among the newer cardiovascular risk markers, which appear to be closely linked to cardiovascular events, in particular sudden cardiac death (10). HRV has also been linked to sudden death syndrome in infants (11). HRV reflects cardiac vagal tone, which is associated with infant maturation and has been suggested to be a developmental expression of the autonomic nervous system (12). HR and HRV have been shown to decrease and increase, respectively, with age during infancy and early childhood (13-15) and to be associated with cognitive function $(12,16,17)$. In observational studies, maternal $n$-3LCPUFA intake or neonatal " $n$-3PUFA-status" have been associated with later cognitive function and problem behavior $(18,19)$. Thus, $n$-3PUFA may affect physiologic as well as cognitive functions.

We performed a $2 \times 2$ randomized trial in 9-mo-old infants to see whether 3 mo of fish oil supplementation affects health status, assessed by HR, HRV, blood pressure, and plasma lipid profile in late infancy. We have previously published the blood pressure and plasma lipid profile results (20), and this article focuses on HR and HRV.

Abbreviations: ANCOVA, analysis of covariance; DHA, docosahexaenoic acid; EPA, eicosapentaenoic acid; FA \% , area percentage of total fatty acids; HR, heart rate; HRV, heart rate variability; $\boldsymbol{n}$-3LCPUFA, long-chain $n$-3 polyunsaturated fatty acids with 20 carbons or more; $\boldsymbol{n}$-3PUFA, polyunsaturated fatty acid of the $n-3$ type; RBC, erythrocyte; SDNN, SD of all RR intervals in the recording 


\section{SUBJECTS AND METHODS}

The study design, recruitment procedure, subjects, and interventions have been reported in detail (20) and are described only briefly. The study had a randomized $2 \times 2$ factorial design, in which infants were randomized to receive fish oil or no supplement from 9 to 12 mo of age. Within each of these groups, the infants were also randomized to drink either standard infant formula or whole cow's milk. Ninety-four infants were recruited from the National Danish Civil Registry during May-October 2003. The inclusion criteria were singleton infants born at $\geq 37$ wk of gestation, birth weight $>2500 \mathrm{~g}$ and above the 5 th percentile for gestational age according to Danish reference material, 5-min Apgar score $\geq 7$, no major complications during pregnancy or at delivery, and no chronic diseases. Only infants with a daily consumption of cow's milk or formula, whose parents agreed to the principle of randomization, were included. If the child was allocated to fish oil, five 105 mL bottles of Eskimo-3 (Cardinova, Sweden; donated by Anjo A/S, Frederiksberg, Denmark) were provided from our department. The parents were asked to give their infant 1-2 teaspoonfuls daily, to keep open bottles refrigerated, to return remaining bottles, and to report waste. The mean intake of fish oil was estimated to be $3.3 \mathrm{~mL} / \mathrm{d}$ (range, $0.8-5.0 \mathrm{~mL} / \mathrm{d}$ ), corresponding to $924 \mathrm{mg} / \mathrm{d} n$-3LCPUFA. The drop out rate after randomization was $12 \%$ $(n=11)$ and not significantly different between the groups $(p=0.149)$.

The study was approved by the Ethical Committee of the Municipalities of Frederiksberg and Copenhagen (J. no. KF 02-014/03) and has been registered in a clinical trial database (ClinicalTrials.gov NCT 00379171). Both parents of all participating infants gave written consent to participate after the study had been explained to them orally and in writing.

The infants were examined at our department before the beginning of the intervention at the age of $9 \mathrm{mo} \pm 3 \mathrm{wk}$ and at the end of the intervention, $3 \mathrm{mo} \pm 3 \mathrm{wk}$ later. Here, the parents were instructed in food recording and interviewed about the diet, growth, and health of the infant. Infant diet was recorded for 7 consecutive $\mathrm{d}$ by use of a precoded dietary record. To standardize fasting, the parents were asked to give a meal approximately $2 \mathrm{~h}$ before the examination. At both visits, we measured blood pressure, HR, and anthropometry and took venous blood samples. The fatty acid composition of the erythrocytes $(\mathrm{RBC})$ was analyzed on $1-\mathrm{mL}$ heparinized blood extracted by the Folch procedure (21) in the presence of butylated hydroxytoluen and analyzed by gas liquid chromatography [HP-6800, Hewlett-Packard, as described in (20)]. RBC fatty acid composition was successfully in around 68\% of the infants at each visit and in $53 \%$ of the infants at both occasions. Values are expressed as the chromatogram area of specific fatty acids relative to the total chromatogram area (FA\%).

Measurement of $\boldsymbol{H R}$ and $\boldsymbol{H R V}$. The HR and HRV were obtained from ECG recordings as previously described (22). In brief, ECGs were recorded continuously for $0.5 \mathrm{~h}$ (including calibration time) by a two-channel tape recorder (Tracker Reynolds, Reynolds Medical, Hertford, UK), while the child was doing an attention task [as described by Colombo et al. (23)]. All recordings were analyzed with commercially available software from Diagnostics Monitoring (Santa Ana, CA). The following time-domain variables were analyzed: the mean length of all normal RR intervals during the recording (RR), the SD of all RR intervals in the recording (SDNN), the mean of the SDs of all normal RR intervals in 5-min segments of the recording, the $\mathrm{SD}$ of the mean of RR intervals measured in successive 5-min intervals, and the square root of the mean of the sum of the squares of the differences between adjacent intervals. Noise and errors were excluded during the editing process and $>80 \%$ of the RR intervals in the ECG recordings had to be present to be included. The assessed HR- and HRV-parameters were based on the basis of $1030 \pm 766$ normal QRS-complexes $(n=114)$. HRV was successfully determined in 84 infants at $9 \mathrm{mo}$, in 61 infants at $12 \mathrm{mo}$, and in 57 infants at both 9 and 12 mo. Only infants with HRV data at both occasions have been included in this article. The characteristics of these infants are shown in Table 1.

Statistics. All data were analyzed with SPSS (version 13.0; SPSS Inc., Chicago, IL) and a significance level of $p<0.05$ was used. Results are given as $\bar{x} \pm \mathrm{SE}$ or SD for the descriptive variables. Normality was checked with histograms and the Shapiro-Wilk test. Comparisons of RBC-EPA values at 9 and 12 mo within each group were performed using a paired $t$ test. The descriptive variables and HR and HRV parameters were entered as outcome variables in two-way analyses of covariance (ANCOVA) with fish oil intervention and milk intervention as fixed factors and baseline values as covariate. Gender (15) was also included as a fixed factor in the analysis. The analysis was performed as full factorial analysis including all factors and covariates and interactions between both interventions and gender. Factors were excluded successively according to $p$-value. The fish oil intervention and baseline values were kept in all models. The milk intervention did not have significant effects in any of the final models, and no significant interaction was seen between the two interventions. Data are therefore presented with the milk
Table 1. Baseline characteristics of the infants in the fish oil intervention groups

\begin{tabular}{lcc}
\hline & Fish oil & No fish oil \\
\hline$n$ & 26 & 31 \\
Gender ( $n, \mathrm{M}: \mathrm{F})$ & $13: 13$ & $15: 16$ \\
Gestational age at delivery (wk) & $40.4 \pm 1.4$ & $40.3 \pm 1.5$ \\
Birth weight (kg) & $3.73 \pm 0.50$ & $3.67 \pm 0.44$ \\
Age (mo) & $9.1 \pm 0.3$ & $9.1 \pm 0.3$ \\
Weight $(\mathrm{kg})$ & $9.28 \pm 0.98$ & $9.14 \pm 0.80$ \\
Length $(\mathrm{cm})$ & $72.5 \pm 2.2$ & $72.2 \pm 2.1$ \\
Maternal fish intake (times/mo) & $4.3(3.8-9.3)$ & $6.5(4.3-15.2)$ \\
Total duration of breast-feeding (mo) & $7.1(4.0-10.0)$ & $9.1(5.9-11.2)$ \\
Energy intake (kJ/d) & $3272 \pm 1067$ & $3614 \pm 1348$ \\
Protein intake (E\%) & $11.6 \pm 1.7$ & $12.1 \pm 1.7$ \\
Carbohydrate intake (E\%) & $50.8 \pm 5.0$ & $50.2 \pm 3.8$ \\
Fat intake (E\%) & $37.6 \pm 4.9$ & $37.7 \pm 4.2$ \\
Intake of linoleic acid (g/d) & $3.0 \pm 1.1$ & $3.0 \pm 1.3$ \\
Intake of $\alpha$-linoleic acid (g/d) & $0.6 \pm 0.2$ & $0.6 \pm 0.2$ \\
Age at introduction of fish (mo) & $7.1 \pm 1.2$ & $6.8 \pm 0.9$ \\
Milk group ( $n$, infant formula:cow's & $16: 10$ & $16: 15$ \\
$\quad$ milk) & &
\end{tabular}

The descriptive data are given as mean $\pm \mathrm{SD}$, ratios or medians $(25-75$ percentile range).

No significant group differences were found in a $t$ test.

groups combined. If a significant interaction was observed between the fish oil intervention and gender, the above analyses were performed in the gender subgroups. Significant differences in HR and HRV between the groups were further investigated by multiple regression analysis to test a possible dosedependent relationship between RBC-EPA or total $n$-3PUFA content [biomarkers of the fish oil intake (20)] and the outcome variables, as described for the ANCOVA above. In correlation analyses with HR-measures at baseline, we used DHA and not EPA, as DHA best reflects habitual $n$-3LCPUFA-intake. Bivariate correlations were performed with Pearson's Product Moment Correlation. With a mean of 28 infants with successful assessments in each group, this study was powered to show an effect of $0.75 \times \mathrm{SD}$ in the different HR-parameters.

\section{RESULTS}

The fatty acid composition of the RBC at baseline did not differ between the two fish oil groups, and fish oil supplementation significantly increased the content of $n$-3LCPUFA in the RBC of the infants with a concomitant decrease in $n-6$ fatty acids (24). Total $n$-3LCPUFA content of the RBC was $14.5 \pm 2.8$ and $8.6 \pm 1.6 \mathrm{FA} \%$ at $12 \mathrm{mo}$ in the fish-oilsupplemented and unsupplemented groups, respectively. The RBC content of $n$-3LCPUFA, especially that of EPA, was linearly associated with the estimated daily fish oil intake ( $r=$ $0.86, n=53, p<0.001)(20)$. RBC-EPA was increased by $2.64 \pm 0.31(n=24, p<0.001)$ FA\% point in the fish-oilsupplemented groups and by only $0.24 \pm 0.06 \mathrm{FA} \%$ point $(n=22, p=0.006)$ in the unsupplemented groups.

Values for the HR and HRV variables at 9 and 12 mo are given in Table 2. A significant association was observed in ANCOVA between the 9 and 12 mo values for all HR and HRV parameters (data not shown). No differences in HRV were observed between the groups in ANCOVAs, but there was an effect of fish oil intervention $\times$ gender on mean RR $(p=0.001)$. The fish oil intervention was found to have an effect on the mean RR interval in boys, whereas no significant difference was seen in girls (Table 3). No significant effect of fish oil intervention $\times$ gender was observed for the other HRV-parameters. The $p$-value for the effect of fish oil in the 
Table 2. Measures of heart rate and heart rate variability before and after the intervention

\begin{tabular}{|c|c|c|c|c|c|c|}
\hline & \multicolumn{3}{|c|}{ Fish oil } & \multicolumn{3}{|c|}{ No fish oil } \\
\hline & $9 \mathrm{mo}$ & $12 \mathrm{mo}$ & Difference & $9 \mathrm{mo}$ & $12 \mathrm{mo}$ & Difference \\
\hline$n$ HRV-parameters & \multicolumn{3}{|c|}{26} & \multicolumn{3}{|c|}{31} \\
\hline Effective ECG-time (min) & $17.0 \pm 1.8$ & $16.5 \pm 0.9$ & - & $16.8 \pm 0.9$ & $15.9 \pm 1.1$ & - \\
\hline $\mathrm{RR}(\mathrm{ms})^{*}$ & $447.1 \pm 6.4$ & $456.4 \pm 6.3$ & $9.3 \pm 7.0$ & $451.2 \pm 5.2$ & $454.6 \pm 4.4$ & $3.4 \pm 5.8$ \\
\hline SDNN (ms) & $27.9 \pm 1.5$ & $29.4 \pm 1.3$ & $1.4 \pm 1.4$ & $28.2 \pm 1.4$ & $27.5 \pm 1.0$ & $-0.7 \pm 1.6$ \\
\hline SDANNi (ms) & $5.9 \pm 0.8$ & $7.9 \pm 1.1$ & $2.0 \pm 1.4$ & $7.4 \pm 1.0$ & $7.1 \pm 0.8$ & $-0.3 \pm 1.3$ \\
\hline SDNNi (ms) & $26.5 \pm 1.4$ & $27.3 \pm 1.2$ & $0.8 \pm 1.2$ & $25.7 \pm 1.3$ & $25.5 \pm 1.0$ & $-0.3 \pm 1.5$ \\
\hline $\operatorname{RMSSD}(\mathrm{ms}) \dagger$ & $14(11-21)$ & $15(12-17)$ & $-0.1 \pm 1.0$ & $16(10-21)$ & $15(12-18)$ & $-0.6 \pm 1.4$ \\
\hline
\end{tabular}

* A sex-treatment interaction was observed for RR $(p=0.001)$, but no significant differences between the fish oil and the no fish oil-group were found by a group comparison of the other variables.

$\dagger$ Not Gauss distributed and thus shown as median (25-75 percentile). All other data are shown as mean \pm SE.

RR, Mean length of all normal RR-intervals during the recording; SDNN, SD of all RR-intervals in the recording; SDANNi, SD of the mean of RR-intervals measured in successive 5-min intervals; SDNNi, mean of the SDs of all normal RR intervals in 5-min segments of the recording; and RMSSD, the square root of the mean of the sum of the squares of the differences between adjacent intervals.

Table 3. Heart rate in gender subgroups before and after the intervention

\begin{tabular}{|c|c|c|c|c|}
\hline \multirow[b]{2}{*}{ Mean \pm SE } & \multicolumn{2}{|c|}{ Fish oil } & \multicolumn{2}{|c|}{ No fish oil } \\
\hline & Girls & Boys & Girls & Boys \\
\hline$n$ & 13 & 13 & 16 & 15 \\
\hline RR 9 mo (ms) & $439.7 \pm 10.7$ & $454.4 \pm 7.0$ & $451.6 \pm 7.7$ & $450.8 \pm 7.1$ \\
\hline RR 12 mo (ms) & $437.8 \pm 7.4$ & $474.9 \pm 7.5$ & $460.1 \pm 7.3$ & $448.8 \pm 4.8$ \\
\hline $\begin{array}{l}\text { Difference in mean RR } \\
9-12 \text { mo }(\mathrm{ms})\end{array}$ & $-1.9 \pm 9.0$ & $20.5 \pm 10.0 *$ & $8.5 \pm 9.4$ & $-2.1 \pm 6.8$ \\
\hline
\end{tabular}

* Different from the boys in the no fish oil-group (ANCOVA $p=0.007$, including baseline and \pm FO). No significant was found between the girls in the fish oil and the no fish oil group.

$\mathrm{RR}$, Mean length of all normal RR-intervals during the recording.

final model for HRV expressed as SDNN was 0.22. No significant group difference was found on the raw HR estimate from the ECG recordings and the $p$-value of the treatment-sex interaction was 0.111 (data not shown).

In infants with successful assessment of both ECG and RBC fatty acid composition at both 9 and 12 mo, the change in mean RR from 9 to 12 mo was strongly associated with the change in RBC-EPA (Fig. 1) and the change in total $n$-3PUFA $(r=0.694, n=30, p<0.001)$. Furthermore, there was a tendency toward an association between mean RR at 9 mo and the total $n$-3PUFA content in RBC at 9 mo $(r=0.281, n=38$, $p=0.088 ; r=0.305, p=0.062$ for DHA). Correlations were observed between the 9- and 12-mo change in mean RR and the changes in $\operatorname{SDNN}(r=0.442, n=57, p=0.001)$ and the other HRV parameters (data not shown). The change in all HRV parameters between 9 and 12 mo was found to be correlated with the baseline values (with $r$-values of 0.6-0.7, $n=57, p<0.001$ )-thus, the higher the variability at baseline, the smaller the potential modification by fish oil.

No significant difference was found in the increase in RBCEPA between the boys and girls in the fish-oil-supplemented groups (data not shown). However, in three of the infants from the fish-oil-supplemented groups, all girls, RBC-EPA was only increased by $0.31-0.55$ FA\% point. The RBC-EPA change in one of these infants was clearly below that expected from the reported intake of fish oil. Information about the intake of fish oil from the other two infants could not be obtained. The RBC-EPA values of these subjects indicate a low actual compliance, which based on the change in RBC-EPA are expected to have been maximally around $0.5 \mathrm{~mL}$ fish oil/d. When an ANCOVA of the effects on mean RR (interventions, gender, baseline) was performed including only infants with a confirmed relevant change in RBC fatty acid composition $(>1 \%$ point EPA increase in the

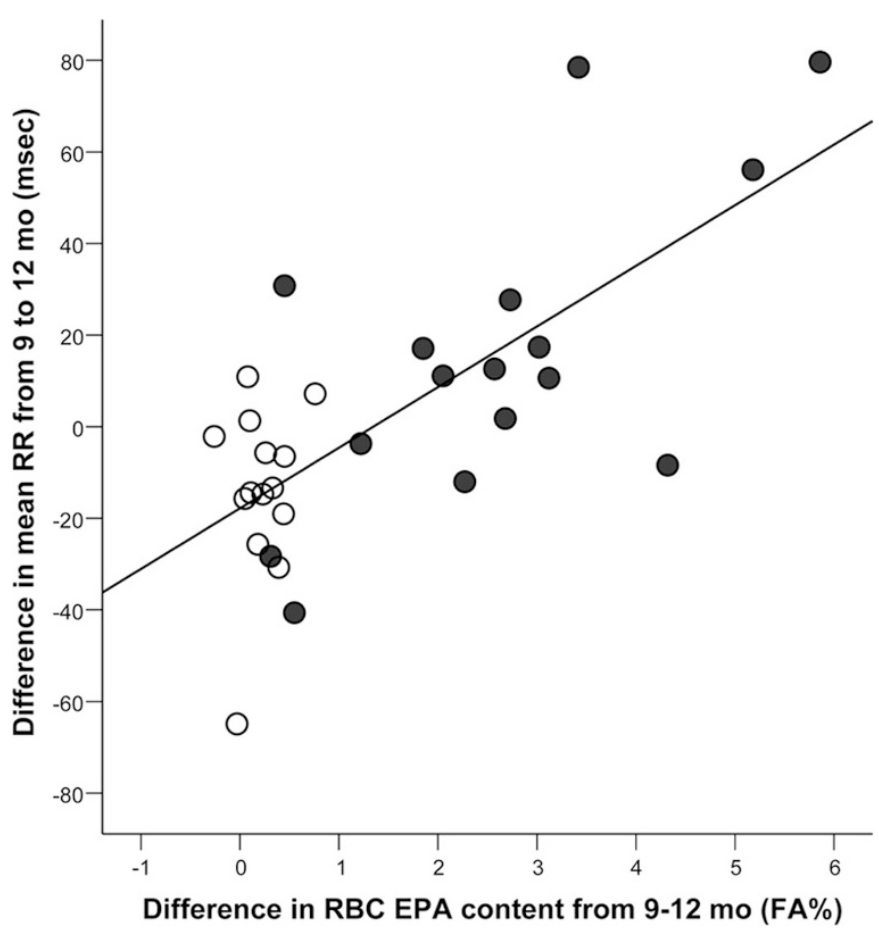

Figure 1. Correlation between the changes from 9 to $12 \mathrm{mo}$ in erythrocytes EPA content and heart rate expressed as RR interval in the fish-oilsupplemented group $(\bullet)$ and the No fish oil control group $(\bigcirc)(r=0.711, p<$ $0.001, n=30$ ). 
fish-oil-supplemented groups), then fish oil was found to have a significant effect on mean RR ( $p=0.011, n=27$ ), whereas gender and fish oil intervention $\times$ gender were no longer statistically significant.

\section{DISCUSSION}

We showed that 3 mo of fish oil supplementation resulted in a pronounced increase in infant RBC $n$-3PUFA, a commonly used biomarker of $n$-3LCPUFA intake. Furthermore, we found that fish oil supplementation resulted in a longer mean RR (lower HR) among boys. This effect on HR was strengthened by a significant correlation between the increase in RBC $n$-3LCPUFA and the change in mean RR during the intervention. Because no gender specificity was found in the correlation, the gender difference appears to be due to differences in compliance between the genders and thus not a true physiologic difference. Therefore, the overall results of the trial may indicate a decrease in HR. Several studies have shown a strong association between HR and HRV in young children (13). This study is, to our knowledge, the first randomized trial to investigate the effect of $n$-3LCPUFA intake on heart rhythm and other cardiovascular risk markers $[$ e.g., blood pressure and plasma lipid profile (20)], in late infancy. Butte et al. (25) have found lower $18 \mathrm{~h}-\mathrm{HR}$ at 1 and $4 \mathrm{mo}$ in breast-fed infants compared with formula-fed infants. This may be due to differences in $n$-3PUFA intake, because formulas at that time contained only $\alpha$-linolenic acid and no $n$-3LCPUFA. In a randomized trial, in which mothers were supplied with fish oil during lactation, we have previously observed a significant gender-specific effect of $n$-3LCPUFA on mean RR interval and HRV in the 2.5-y-old children, with lower HR in boys, but higher HR and lower HRV in girls (22).

The infants in the present study had higher HR and lower HRV than we have previously observed in 2.5-y-old children measured under similar conditions (22). This is accordance with what we expected due to the difference in age $(13,14)$. The infant HRV measures in the present study were lower than those reported in studies with 18-24 h ECG recordings, but the RR intervals were comparable to those observed in other studies during late infancy $(13,14,25)$. No effect of the fish oil was detected on the raw HR estimates, but calculation of the mean RR interval provides the most valid assessment of HR. The short ECG recordings may be a limitation in the present study and they were furthermore not made under strictly standardized circumstances. We tried to plan the visits so that the infant was awake and not hungry, but fasted for preferably $2 \mathrm{~h}$. A more complete standardization would be hard to achieve in healthy young children. The ECG was recorded while the infants were doing an attention task, which may have influenced the HR and HRV and may have promoted differences between the groups. This trial only included a small group of children and is presumed to have had relatively high levels of random variation on the outcome measurements. Sample size was unfortunately reduced because of a low success rate of the ECG recordings (only about half of the infants on both visits). The power of the trial is therefore low, thus increasing the probability of not detecting actual effects (type II errors). The power of the gender subgroup analysis was even lower, but the indicated effect in boys and the dose-response relationship of fish oil on mean RR, as well as the effects on blood pressure and plasma lipid profile (20), were in agreement with results from larger more controlled adult trials.

We wanted to study possible effects of the traditional Nordic habit of giving fish oil to infants so no placebo was used as a control to the fish oil supplement. The infants were given fish oil doses comparable with that used in the many adult studies. The dose was comparable with that recommended in Norway from 1 mo of age and thus assumed to be safe, but no safety measures such as bleeding time were included. Based on the RBC fatty acid composition, there appears to be some lack of compliance and an unreliable parental reporting of compliance. Thus, we assumed a similar low compliance in the children from whom we could not verify this due to lack of blood samples. A major strength of the current study is the inclusion of blood samples from the infants, because this is allowed for research purposes in Denmark. However, the unsuccessful blood sampling resulted in only approximately half of the infants having both samples, but no significant difference was observed between the change in RR from 9 to 12 mo in infants with or without blood samples (data not shown). Although not significant, the total duration of breast feeding and maternal fish intake was low in the fish oil group versus the control group, and this may have blunted the observed difference between the outcomes in the groups.

Despite the limitations, the results from our trial indicate that an increased intake of $n$-3LCPUFA could affect heart rhythm in healthy Danish infants with a habitual fish intake of around $7 \mathrm{~g} / \mathrm{d}$ (20). Several cardiovascular risk markers, including HRV, appear to be most pronouncedly affected by fish oil in individuals who are older, have a less healthy risk profile or a low content of $n$-3PUFA in cell membranes from various tissues $(2,3,26,27)$. The level of RBC-DHA of the infants in this study was comparable with those previously found in 2.5-y-old Danish children, which was decreased compared with that in early infancy and in mothers during pregnancy and lactation $(28,29)$. The fact that we detected physiologic effects of the fish oil supplement, together with the low RBC-DHA level could indicate that healthy Danish infants may have a low " $n$-3PUFA-status." However, the $n$-3LCPUFA intake in Denmark is relatively high compared with many other countries, probably due to a high intake of fatty fish such as mackerel and herring, which is substantiated by a considerably higher content of DHA in breast milk from Danish mothers, compared with other Western countries $(1,30)$. The " $n$-3PUFA-status" in Danish infants also seems to be more favorable compared with other populations. The $n$-3LCPUFA content of RBC at 12 mo in our unsupplemented group (24) was comparable with the levels found in the groups supplemented with DHA-enriched eggs in two studies from the United States and Australia $(31,32)$. Thus, we would expect that the results we report here would be stronger in infants from countries with a lower fish intake.

The acute and long-term clinical consequences of a slightly lower HR or higher HRV in infancy are, to our knowledge, not known. A cross-sectional study has demonstrated that higher 
HR is related to less s.c. fat, but the causality is not known (33). It is also not known to what extend HR is tracking from childhood to adulthood. Tracking may be caused by biologic programming or a tracking in lifestyle and lifestyle markers, e.g., diet has been shown to "track" from adolescence to adulthood (34). Dietary effects on HR may be caused by changes in cardiac electrophysiology or changes in the autonomic control of the heart (35). An effect of $n$-3PUFA on the autonomic control of heart rhythm may provide a common link between possible effects on HR in infants and on CNS development. The observed effect of fish oil, especially in the boys in this study, may be interpreted as an indicator of accelerated CNS maturation, because vagal tone increases with age, as reflected by a decreasing HR and an increase in HRV during early childhood. Increased vagal tone has been shown to be associated with cognitive function (12). Neonatal cardiac vagal tone during sleep in very low weight infants has also been found to be associated with school-age social competence (16). Vagal tone-typically assessed during sleep in infants - has also been suggested as a physiologic marker of stress vulnerability (12). Vagal tone changes in response to stressors, such as mental demands (12) and changes in vagal tone during an attention tasks at 9 mo of age has been observed to be closely associated with behavioral problems later in childhood (17). Maternal fish intake and early infant " $n$-3PUFA-status" has also been associated with later problem behavior in observational studies $(18,19)$.

This study suggests that fish oil supplementation has an effect on HR in healthy Danish infants similar to that in adults. The immediate effects of a HR-modulation on infant health are uncertain. The indicated effect of fish oil on HR, especially in boys, may imply low " $n$-3PUFA-status" in late infancy and differences in CNS function. As the low intake during late infancy also could have negative effects on cognitive function. It is of importance to further explore whether an increased $n$-3LCPUFA intake during the complementary feeding period could have beneficial long-term effects.

Acknowledgments. We gratefully acknowledge the contribution of all the participating children and their families. We are also grateful to Majken Ege Rasmussen, Vivian Julia Anker, Susan Bjerregaard Nielsen, and Puk Maja Ingeman Holm for assisting in the collection of data; to technician Grete Peitersen for doing the fatty acid analysis; and to technician Rikke Bülow Eschen, who performed the HRV analysis.

\section{REFERENCES}

1. Lauritzen L, Hansen HS, Jørgensen MH, Michaelsen KF 2001 The essentiality of long-chain $n-3$ fatty acids in relation to development and function of the brain and retina. Prog Lipid Res 40:1-94

2. Geleijnse JM, Giltay EJ, Grobbee DE, Donders AR, Kok FJ 2002 Blood pressure response to fish oil supplementation: metaregression analysis of randomized trials. J Hypertens 20:1493-1499

3. Mozaffarian D, Geelen A, Brouwer IA, Geleijnse JM, Zock PL, Katan MB 2005 Effect of fish oil on heart rate in humans-a meta-analysis of randomized controlled trials. Circulation 112:1945-1952

4. Das UN 2003 A perinatal strategy to prevent coronary heart disease. Nutrition 19:1022-1027

5. Armitage JA, Khan IY, Taylor PD, Nathanielsz PW, Poston L 2004 Developmental programming of the metabolic syndrome by maternal nutritional imbalance: how strong is the evidence from experimental models in mammals? J Physiol 561:355-377
6. Armitage JA, Pearce AD, Sinclair AJ, Vingrys AJ, Weisinger RS, Weisinger HS 2003 Increased blood pressure later in life may be associated with perinatal $n-3$ fatty acid deficiency. Lipids 38:459-464

7. Forsyth JS, Willatts P, Agostoni C, Bissenden J, Casaer P, Boehm G 2003 Long chain polyunsaturated fatty acid supplementation in infant formula and blood pressure in later childhood: follow up of a randomised controlled trial. BMJ 326:953

8. Agostoni C, Decsi T, Fewtrell M, Goulet O, Kolacek S, Koletzko B, Michaelsen KF, Moreno L, Puntis J, Rigo J, Shamir R, Szajewska H, Turck D, van Goudoever J 2008 Complementary feeding: a commentary by the ESPGHAN Commitee on nutrition. J Pediatr Gastroenterol Nutr 46:99-110

9. Schmidt EB, Arnesen H, Christensen JH, Rasmussen LH, Kristensen SD, De Caterina R 2005 Marine $n-3$ polyunsaturated fatty acids and coronary heart disease: Part II: Clinical trials and recommendations. Thromb Res 115:257-262

10. Task Force of the European Society of Cardiology and the North American Society of Pacing and Electrophysiology. 1996 Heart rate variability: standards of measurement, physiological interpretation, and clinical use. Circulation 93:1043-1065

11. Horne RS 2006 Effects of prematurity on heart rate control: implications for sudden infant death syndrome. Expert Rev Cardiovasc Ther 4:335-343

12. Porges SW 1992 Vagal tone: A physiological marker of stress vulnerability. Pediatrics 90:498-504

13. Massin M, vonBernuth $\mathrm{G} 1997$ Normal ranges of heart rate variability during infancy and childhood. Pediatr Cardiol 18:297-302

14. Finley JP, Nugent ST 1995 Heart-rate-variability in infants, children and youngadults. J Auton Nerv Syst 51:103-108

15. Silvetti MS, Drago F, Ragonese P 2001 Heart rate variability in healthy children and adolescents is partially related to age and gender. Int J Cardiol 81:169-174

16. Doussard-Roosevelt JA, McClenny BD, Porges SW 2001 Neonatal cardiac vagal tone and school-age developmental outcome in very low birth weight infants. Dev Psychobiol 38:56-66

17. Porges SW, Doussard-Roosevelt JA, Portales AL, Greenspan SI 1996 Infant regulation of the vagal "brake" predicts child behavior problems: a psychobiological model for social behavior. Dev Psychobiol 29:697-712

18. Hibbeln JR, Davis JM, Steer C, Emmett P, Rogers I, Williams C, Golding J 2007 Maternal seafood consumption in pregnancy and neurodevelopmental outcomes in childhood (ALSPAC study): an observational cohort study. Lancet 369:578-585

19. Krabbendam L, Bakker E, Hornstra G, van Os J 2007 Relationship between DHA status at birth and child problem behaviour at 7 years of age. Prostaglandins Leukot Essent Fatty Acids 76:29-34

20. Damsgaard CT, Schack-Nielsen L, Michaelsen KF, Fruekilde MB, Hels O, Lauritzen L 2006 Fish oil affects blood pressure and the plasma lipid profile in healthy Danish infants. J Nutr 136:94-99

21. Folch J, Lees M, Sloane Stanley GH 1957 A simple method for the isolation and purification of total lipids from animal tissues. J Biol Chem 226:497-509

22. Larnkjær A, Christensen JH, Michaelsen KF, Lauritzen L 2006 Blood pressure and heart rate variability in children 2 years after fish oil supplementation of their lactating mothers. J Nutr 136:1539-1544

23. Colombo J, Kannass KN, Shaddy DJ, Kundurthi S, Maikranz JM, Anderson CJ, Blaga OM, Carlson SE 2004 Maternal DHA and the development of attention in infancy and toddlerhood. Child Dev 75:1254-1267

24. Damsgaard CT, Lauritzen L, Kjaer TM, Holm PM, Fruekilde MB, Michaelsen KF, Frokiaer H 2007 Fish oil supplementation modulates immune function in healthy infants. J Nutr 137:1031-1036

25. Butte NF, Smith EO, Garza C 1991 Heart rates of breast-fed and formula-fed infants. J Pediatr Gastroenterol Nutr 13:391-396

26. Rees D, Miles EA, Banerjee T, Wells SJ, Roynette CE, Wahle KW, Calder PC 2006 Dose-related effects of eicosapentaenoic acid on innate immune function in healthy humans: a comparison of young and older men. Am J Clin Nutr 83:331-342

27. Christensen JH, Christensen MS, Dyerberg J, Schmidt EB 1999 Heart rate variability and fatty acid content of blood cell membranes: a dose-response study with n-3 fatty acids. Am J Clin Nutr 70:331-337

28. Lauritzen L, Hoppe C, Straarup EM, Michaelsen KF 2005 Maternal fish oil supplementation in lactation and growth during the first 2.5 years of life. Pediatr Res 58:235-242

29. Lauritzen L, Kjær TM, Fruekilde MB, Michaelsen KF, Frøkiær H 2005 Fish oil supplementation of lactating mothers affects cytokine production in $2 \frac{1}{2}$-year-old children. Lipids 40:669-676

30. Brenna JT, Varamini B, Jensen RG, Diersen-Schade DA, Boettcher JA, Arterburn LM 2007 Docosahexaenoic and arachidonic acid concentrations in human breast milk worldwide. Am J Clin Nutr 85:1457-1464

31. Makrides M, Hawkes JS, Neumann MA, Gibson RA 2002 Nutritional effect of including egg yolk in the weaning diet of breast-fed and formula-fed infants: a randomized controlled trial. Am J Clin Nutr 75:1084-1092

32. Hoffman DR, Theuer RC, Castaneda YS, Wheaton DH, Bosworth RG, O'Connor AR, Morale SE, Wiedemann LE, Birch EE 2004 Maturation of visual acuity is accelerated in breast-fed term infants fed baby food containing dha-enriched egg yolk. J Nutr 134:2307-2313

33. Pereira GR, Johnston FE, McKinney S, Coleman JR, Kurtz J, Horowitz MP, Rounds JL 1984 Heart rate and sleep time: their relationship with growth and adiposity in male infants. J Pediatr Gastroenterol Nutr 3:759-764

34. Lake AA, Mathers JC, Rugg-Gunn AJ, Adamson AJ 2006 Longitudinal change in food habits between adolescence (11-12 years) and adulthood (32-33 years): the ASH30 Study. J Public Health (Oxf) 28:10-16

35. Christensen JH 2003 n-3 fatty acids and the risk of sudden cardiac death. Emphasis on heart rate variability. Dan Med Bull 50:347-367 